\title{
Nonlocal effects in the optical response of composite materials with metallic nanoparticles
}

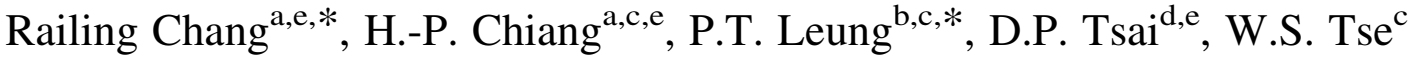 \\ anstitute of Optoelectronic Sciences, National Taiwan Ocean University, 2, Pei-Ning Road, Keelung 20224, Taiwan, ROC \\ ${ }^{\mathrm{b}}$ Department of Physics, Portland State University, P.O. Box 751, Portland, OR 97207-0751, USA \\ ${ }^{\mathrm{c}}$ Institute of Physics, Academia Sinica, Taipei, Taiwan, ROC \\ ${ }^{\mathrm{d}}$ Department of Physics, National Taiwan University, Taipei 10617, Taiwan, ROC \\ ${ }^{\mathrm{e}}$ Center of Nanostorage Research, National Taiwan University, Taipei 10617, Taiwan, ROC
}

Received 13 July 2004; received in revised form 30 September 2004; accepted 6 November 2004 by S.G. Louie

Available online 23 November 2004

\begin{abstract}
A phenomenological model for the optical response of composite materials with metallic nanoparticles is presented. This model applies the conventional effective medium theories (EMT) but takes into account the spatial dispersion effects in the dielectric response of the metallic nanoparticles. This leads to an EMT that depends on the size of the particles. Numerical results from a model computation shows that this effect due to the nonlocal optical response of the nanoparticles can increase the resonant absorption frequency of the composite significantly for particles of very small sizes; and can lead to resonant absorption even in the Bruggeman symmetric EMT - a feature which is believed to be absent in the conventional treatment where local response for the metal particles has been assumed.
\end{abstract}

(C) 2004 Elsevier Ltd. All rights reserved.

PACS: 78.20.Ci; 61.46.+w;

Keywords: A. Disordered systems; D. Optical properties

\section{Introduction}

The theoretical study of the optical/dielectric properties of composite materials containing tiny metallic particles embedded in a dielectric host, such as cermet, is over a century old. Since the first publication of various effective medium theories (EMT) at the beginning of the twentieth century [1,2], there have been many extensions and modifications proposed in the literature over the last thirty years [3]. These include, for example: detailed treatment of

\footnotetext{
* Corresponding authors. Address: Institute of Optoelectronic Sciences, National Taiwan Ocean University, 2, Pei-Ning Road, Keelung 20224, Taiwan, ROC. Tel.: + $86224622192 \times 6714$; fax: + 886224634360 .

E-mail address: rlchang@mail.ntou.edu.tw (R. Chang).
}

the particle shape beyond that of a sphere [4,5]; consideration of the possibility of particle coalescence [5,6]; account for particle correlation through multiple scattering effects [7]; improved treatment of percolation threshold in these models [8]; generalization to an anisotropic effective dielectric response for the composite [9]; implication of the significance of multipolar response which is neglected in the conventional EMT [10]; and the various generalizations to include electrodynamic effects leading to an EMT which depends on the sizes of the embedded particles [11].

Particle-size dependent EMT's for composite materials are of considerable interest. The one emerging from electrodynamic effects mentioned above is significant when the particle size $a$ is large compared to the optical wavelength $\lambda$ under consideration. For metallic nanoparticles with sizes much smaller then $\lambda$ in the visible and IR 
regime, a different kind of size effect becomes significant which has to do with the wave nature of the metallic electrons. While in the literature, this quantum size effect has been studied in the context of dielectric response for nanoparticles [12] and composites with such particles [7], it is the purpose of the present work to study a different kind of size effect which arises from the increasing significance of the particle surface. Due to the simple fact that the surfaceto-volume ratio $(\sim 1 / a)$ of the particle increases as the size decreases, the nonlocal optical effects become highly significant for nanoparticles. Here nonlocal effect refers to the wave vector dependence of the dielectric constant $\varepsilon(\vec{k}$, $\omega)$ leading, ideally, to smoothly continuous charge and field distributions across the geometric boundary of the particle. To our knowledge, none of the previous works has devoted to the study of this effect since they have all assumed a dielectric function of the form $\varepsilon(\omega)$ for the particles in which spatial dispersion is ignored. Note that this effect also differs from the one originated from scattering of the free electrons at particle boundaries $[12,13]$, which also leads to a sizedependent EMT. We should point out that in a series of works on the study of electron energy loss spectroscopy
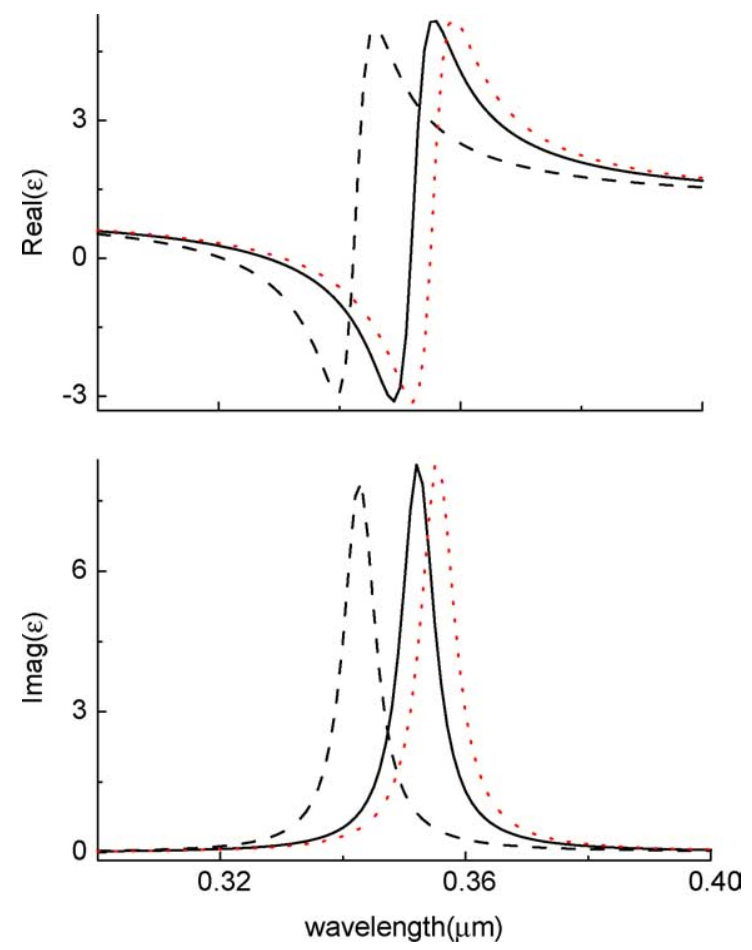

Fig. 1. Plot of the complex dielectric function as a function of wavelength, according to the Maxwell-Garnet theory for a composite with a concentration of metal particle $f=0.05$. The host is assumed vacuum for simplicity, and the Drude parameters are used as in Ref. [20]. The local response (Drude) is plotted in dotted line, and the nonlocal response according to the hydrodynamic model is plotted in solid line (for particle size $a=25 \mathrm{~nm}$ ) and in dashed line (for $a=5 \mathrm{~nm}$ ).
(EELS) from composite materials [14-16], Fuchs and collaborators have studied a different kind of nonlocal effects which arise from inter-particle interaction/correlation among the particles in the composite. The dielectric function for the individual particle is still a local one. As discussed in Ref. [14], these effects studied by Fuchs et al. are insignificant for optical experiments.

In the following, we shall apply a simple phenomenological model for the dielectric response of a sphere due first to Fuchs and Claro [17] to study these nonlocal effects in a composite as described above.

\section{Theoretical model}

For simplicity, we shall assume that in the case of local electrodynamics, the dielectric response for the metallic particles is described by the Drude model:

$\varepsilon(\omega)=1-\frac{\omega_{\mathrm{p}}^{2}}{\omega(\omega+\mathrm{i} \gamma)}$

In this case, it is well-known that $\varepsilon(\omega)$ is independent of the size of the particle and is the same for all multipolar responses. In the case of nonlocal dielectric response, however, this is no longer true and $\varepsilon(\vec{k}, \omega)$ depends on both the particle size and the multipole order of the response in general. Following the theories of Fuchs and coworkers, an 'effective local dielectric function' can be obtained for a sphere using the 'semi-classical infinite barrier' (SCIB) approximation as follows [17]:

$\xi_{\ell}(\omega)=\left[\frac{2}{\pi}(2 \ell+1) a \int_{0}^{\infty} \frac{j_{\ell}^{2}(k a)}{\varepsilon(k, \omega)} \mathrm{d} k\right]^{-1}$

where $\ell$ is the order of the multipolar response, $a$ the radius of the sphere, $j_{\ell}$ the spherical Bessel function, and $\varepsilon(k, \omega)$ the isotropic nonlocal dielectric function which will incorporate certain quantum effects of the electrons in the metal sphere depending on the model adapted [18]. In the following, we shall use Eq. (2) to study the size effect due to the increasing significance of the surface response from the nanoparticles, and shall explore the implication of this effect on the comparison between theory and experiment using various models for the EMT in the literature.

To study the effects of Eq. (2) in composite materials with metallic nanoparticles, we shall adopt both the Maxwell-Garnett (MG) [1] and the Bruggeman (BG) [2] models. According to these, we have the effective dielectric function $\bar{\varepsilon}$ for the composite given implicitly through the following algebraic equations:

$\frac{\bar{\varepsilon}_{\mathrm{MG}}-\varepsilon^{\prime}}{\bar{\varepsilon}_{\mathrm{MG}}+2 \varepsilon^{\prime}}=f \frac{\varepsilon-\varepsilon^{\prime}}{\varepsilon+2 \varepsilon^{\prime}}$

$\frac{f\left(\varepsilon-\bar{\varepsilon}_{\mathrm{BG}}\right)}{\varepsilon+2 \bar{\varepsilon}_{\mathrm{BG}}}=\frac{(f-1)\left(\varepsilon^{\prime}-\bar{\varepsilon}_{\mathrm{BG}}\right)}{\varepsilon^{\prime}+2 \bar{\varepsilon}_{\mathrm{BG}}}$ 
In the above equations, $f$ is the volume fraction of the metal particles, $\varepsilon$ and $\varepsilon^{\prime}$ are the dielectric constants of the metal and the host material, respectively. We shall study the nonlocal optical effects for the composite material by using Eq. (2) for $\varepsilon$, and compared with those from using Eq. (1). Since only the dipolar response of the metal particles is considered in the models in Eqs. (3) and (4), one simply has to substitute $\varepsilon$ by $\xi_{1}$ (i.e. $\xi_{\ell}$ in Eq. (2) with $\ell=1$ ) into them and compare the results from using Eq. (1) for the metallic dielectric function. As for the nonlocal response, we shall use both the hydrodynamic model and the Lindhard-Mermin (RPA) model for $\varepsilon(k, \omega)$ in Eq. (2), in the same way as done in Ref. [17] where the multipolar polarizability of these metal particles was studied.

\section{Numerical results}

To illustrate the aforementioned nonlocal effects in composite materials, we refer to the pioneering works of Abeles and coworkers [4,19,20] which are often cited [21] as one of the earlier works in elucidating various EMT's (e.g. the Maxwell-Garnett (MG) and the Bruggeman (BG) theories) via comparison with experimental results. Fig. 1 shows the complex dielectric function of a composite with low metallic concentration $(f=0.05)$ according to the MG theory and the hydrodynamic $(\mathrm{H})$ model for the nonlocal metallic response. The parameters for the host material and
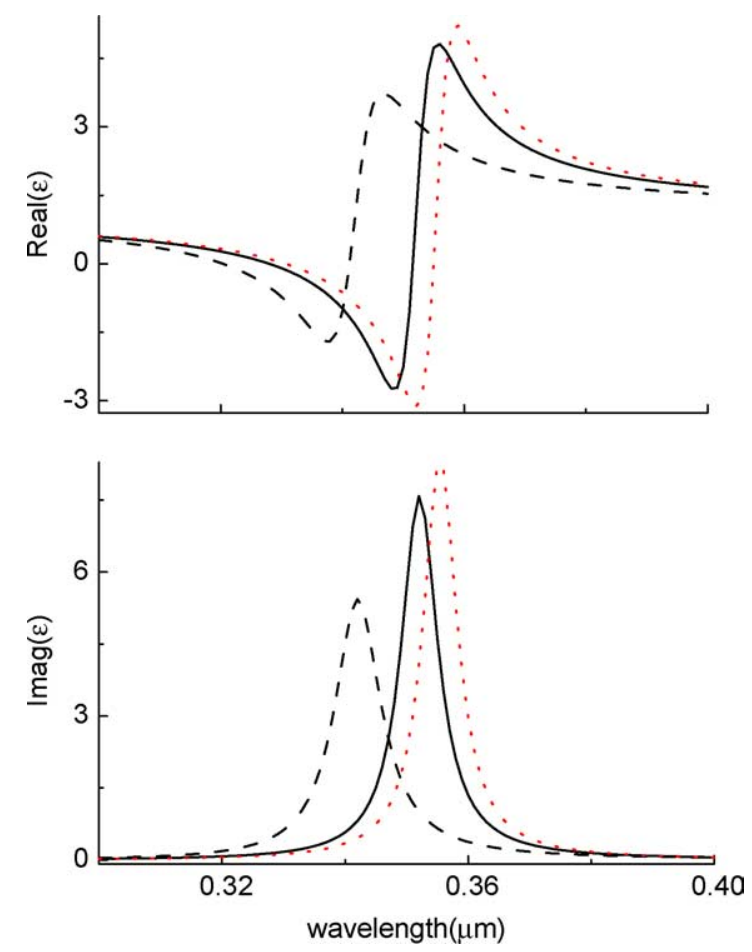

Fig. 2. Same as in Fig. 1, except that the nonlocal results are those from using the Lindhard-Mermin dielectric function. the local (Drude) dielectric function are taken from Ref. [20], with $\omega_{\mathrm{p}}=9.4 \times 10^{15} \mathrm{~s}^{-1}$ and $\gamma=1.0 \times 10^{14} \mathrm{~s}^{-1}$. While it is well-known that the resonance frequency predicted by the MG theory is in general red-shifted from the one for the bulk metal, it is observed that the nonlocal effects will 'blue-shift' the resonance back appreciably for very small metal particles $(a=5 \mathrm{~nm})$. This blue-shift of the dipolar surface plasmon frequency $\left(\omega_{\mathrm{sp}}\right)$ for the nanoparticles has been well-established in the nonlocal theories based on the SCIB formulation [22]. The physical origin of the phenomenon arises from the $k$-dependence of $\omega_{\text {sp }}$, and the fact that this dependence becomes more significant as the particle size decreases. It turns out that this $k$-dependence of $\omega_{\text {sp }}$ can be related to that of the bulk plasmon modes whose dispersion relation has the frequency to increase with $k$. Fig. 2 presents a calculation similar to that in Fig. 1 except that the nonlocal response of the metal is now described by the Lindhard-Mermin (LM) theory. This RPA theory is believed to be more accurate for it accounts not only for the collective plasmon excitation, but also the single-particle ( $\mathrm{e}-\mathrm{h}$ pair) excitation of the free electrons in the metal [18]. The results show that while the resonance frequencies in both the $\mathrm{H}$ and the LM models are pretty much the same, the LM model leads to smaller absolute values for both the real and the imaginary parts of the dielectric functions. Note that the closeness in resonance
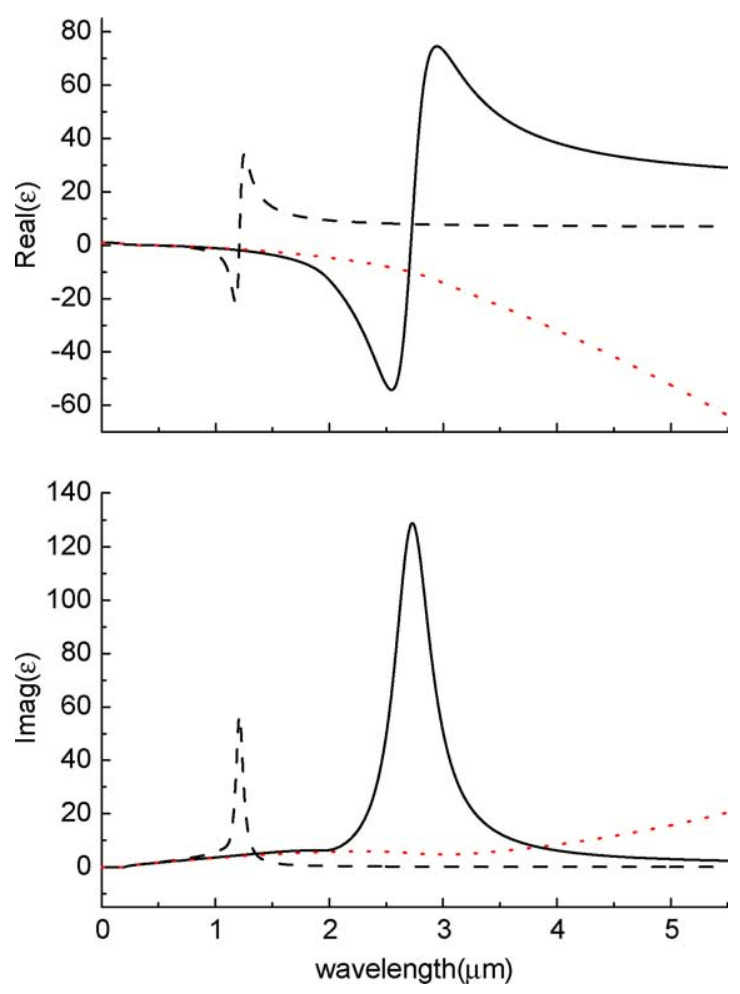

Fig. 3. Similar plot as in Fig. 1, but for a composite with higher metallic concentration $(f=0.4)$ and the Bruggeman EMT has been applied. 
frequency between the two models occurs since we only consider the dipolar $(l=1)$ response in the metal particles [17].

Figs. 3 and 4 show similar calculations as in Figs. 1 and 2 , but for a higher metallic concentration $(f=0.4)$ where the Bruggeman EMT has been applied to compute the effective dielectric function for the composite. While the sizedependent effects due to nonlocal optical response of the metal particles are seen pretty similar to the case with low metallic concentration described by the MG theory (e.g. the LM model leads to smaller values but similar resonance frequencies compared to those from the $\mathrm{H}$ model;... etc.); the most dramatic result in this case is that this nonlocal effect yields a size-dependent absorption resonance frequency in the dielectric function of the composite-which is known to be absent in the Bruggeman theory for composite dielectric functions $[4,19,20]$. In addition, it is seen the difference in absolute values for the dielectric functions between the $\mathrm{H}$ and LM models is more significant in the case with higher metallic concentration as shown in Figs. 3 and 4, with the 'widths' in the imaginary part much broader in the LM case due to the inclusion of both the collective and single-particle excitations of the free electrons.

We speculate that the disappearance and the present reappearance of the resonant absorption frequency in the Bruggeman theory has to do with the percolation threshold
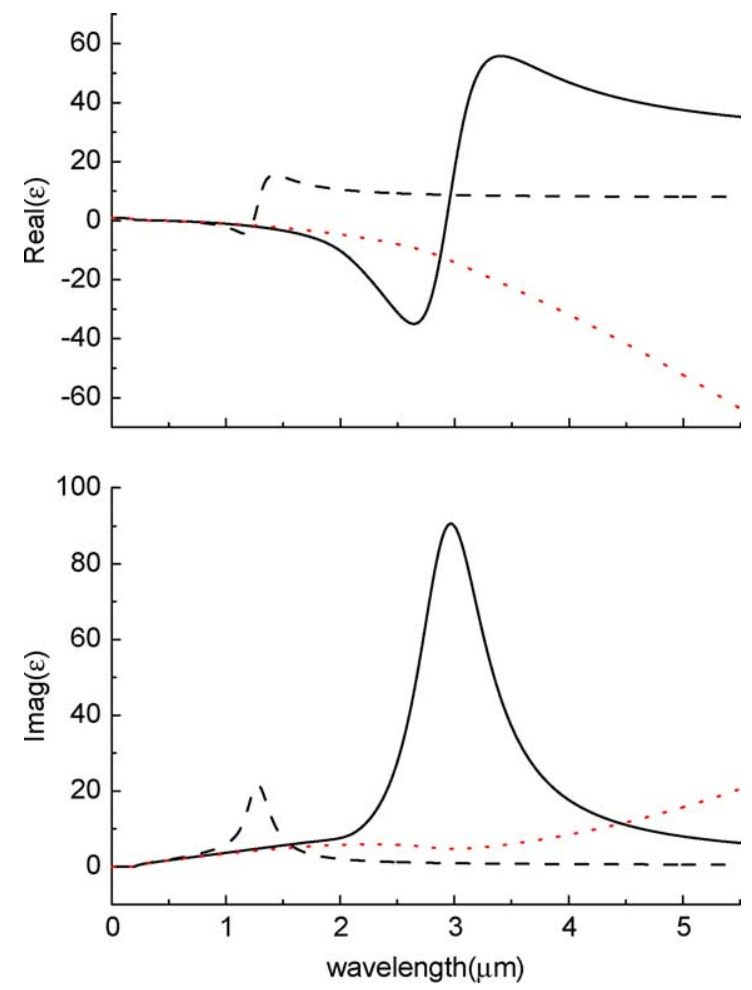

Fig. 4. Same as in Fig. 3, except that the nonlocal results are those from using the Lindhard-Mermin dielectric function. which exists only in the Bruggeman theory but not in the MG theory. It is well-known that when the volume fraction $(f)$ of the metal particles increases beyond a critical value of $1 / 3$, the Bruggeman theory predicts a metallic transition for the composite material; whereas there is no such transition for any value of $f$ in the MG theory [3]. To illustrate this, we have plotted in Figs. 5 and 6 the imaginary part of the composite dielectric function over a large range of $f$ values according to both the MG and Bruggeman theories, with both local and nonlocal results shown according to the hydrodynamic model. It is clear from Fig. 5 that the resonant absorption frequency due to localized surface plasmons (LSP) of the particles always exists in the MG theory, in both the local and nonlocal cases, for all the values of $f$. For small values of $f$, these frequencies are close to the singleparticle LSP frequency [23]. As $f$ increases, the interaction among the metal particles leads to a resonance at longer wavelengths. The nonlocal theory [Fig. 5(b)] simply blueshifts the peaks as explained before. In contrary, in the Bruggeman theory, we see in Fig. 6(a) that the LSP peak starts to be broadened significantly in the usual local theory, due again to the inter-particle interactions. When $f$ exceeds $1 / 3$, an extra resonance takes place towards the zero frequency end (i.e. the well-known 'Drude peak' [23]),
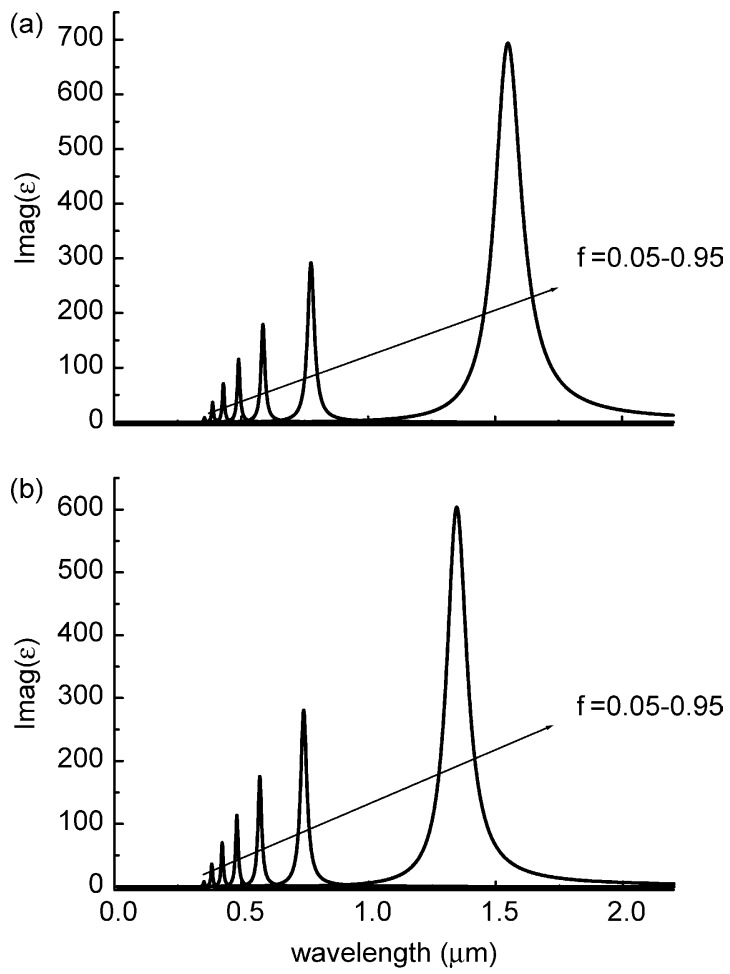

Fig. 5. Plot of the imaginary part of the composite dielectric function according to the Maxwell-Garnet theory as a function of wavelength, over a large range of volume fraction values, according to (a) the local response and (b) the nonlocal response for particle size $a=25 \mathrm{~nm}$. 

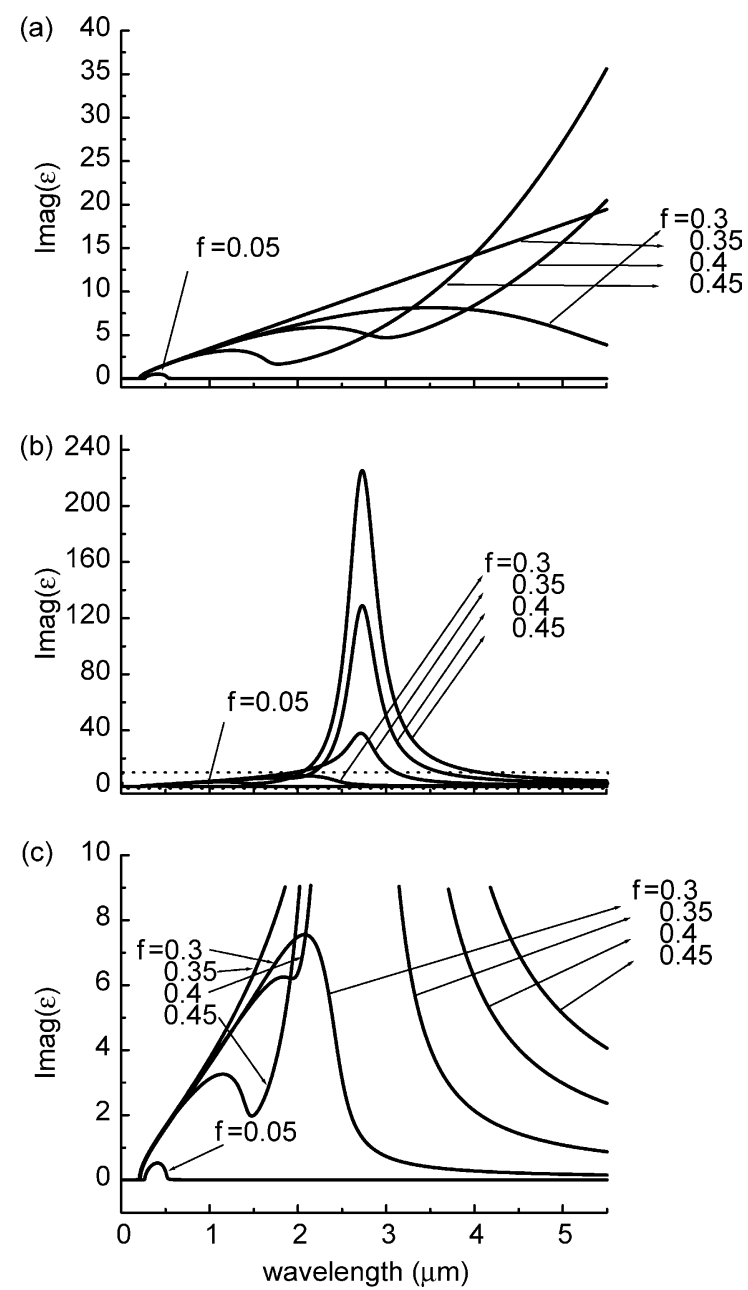

Fig. 6. (a) and (b) Similar to Fig. 5, but for the Bruggeman EMT. (c) The magnification of the part enclosed by dotted line in $6(\mathrm{~b})$.

with the original LSP resonance gradually disappears for larger values of $f$. This we interpret as a 'de-localization' phenomenon of the LSP peak due to percolation in the Bruggeman theory which leads to metallic transition. However, once the particle response becomes nonlocal, we see in Fig. 6(b) and (c) that the Drude peak does no longer exist, and instead a new strong absorption peak appears at a fixed long wavelength of about $2.7 \mu \mathrm{m}$, almost independent of the concentration of the metal particles. It is obvious that this deviation from the 'Drude behavior' after the percolation transition is caused by the nonlocal dielectric response of the metal particles, which, as a result, leads to an extra resonance structure on top of the broadened LSP peak. It will be of interest to study further quantitatively how such long-wavelength resonance depends on the various nonlocal models.

To further demonstrate this 'size-dependent' effect, we have plotted the composite dielectric function as a function of the metal particle size for fixed concentration and wavelength in Figs. 7 and 8, respectively. It is seen that while the Drude model is of course independent of the particle size, both the nonlocal models $\mathrm{H}$ and LM give very different results for small particle sizes. It is also seen that the difference between the two nonlocal models disappear for large particles, a phenomenon due possibly to the dominance of the collective over the single-particle excitation for large particles. Furthermore, it is observed, as expected, that these nonlocal effects will disappear as the particle size increases. This is particularly obvious in the Bruggeman model calculation (with large metal concentration) as shown in Fig. 8.

\section{Conclusion}

In this communication, we have studied a new type particle size-dependent effect in the dielectric response of composite materials. Unlike the other size-dependent effects which have been studied previously in the literature, such as the multipolar electrodynamic effect [11], the quantum size
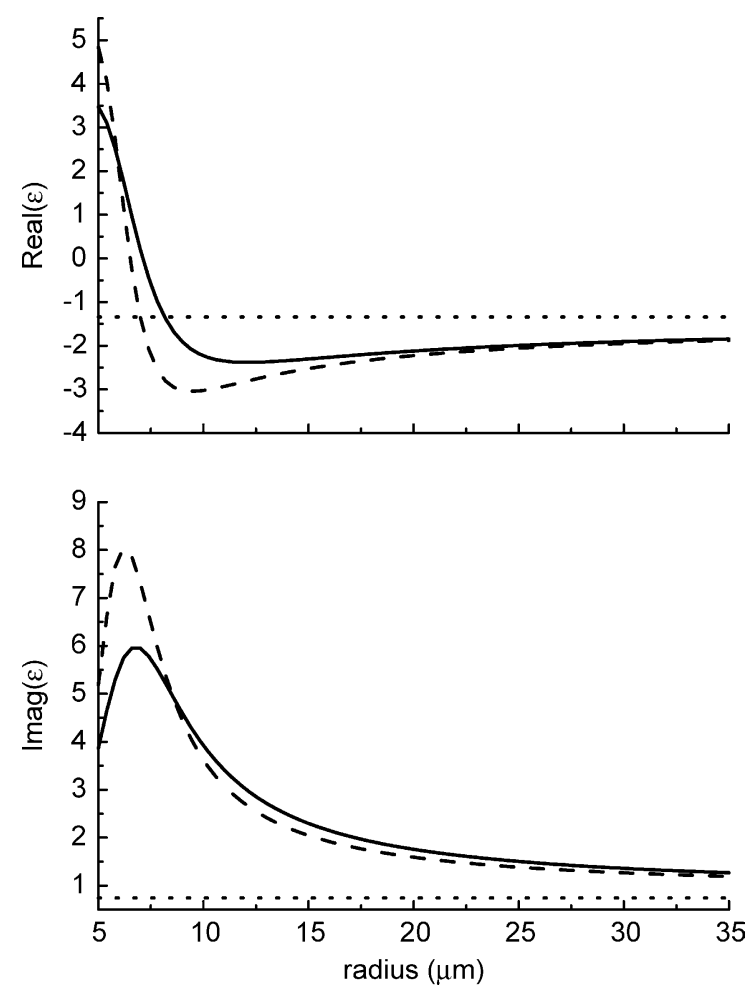

Fig. 7. Plot of the composite dielectric function as a function of the radius of the metal particles at a fixed wavelength $(\lambda=0.345 \mu \mathrm{m})$ and low metal concentration $(f=0.05)$. Note that the MaxwellGarnet theory has been applied. The nonlocal results are represented by the solid curve (from the Lindhard-Mermin model), and by the dashed curve (from the hydrodynamic model); while the local (Drude) result is a constant and is shown as the dotted line. 

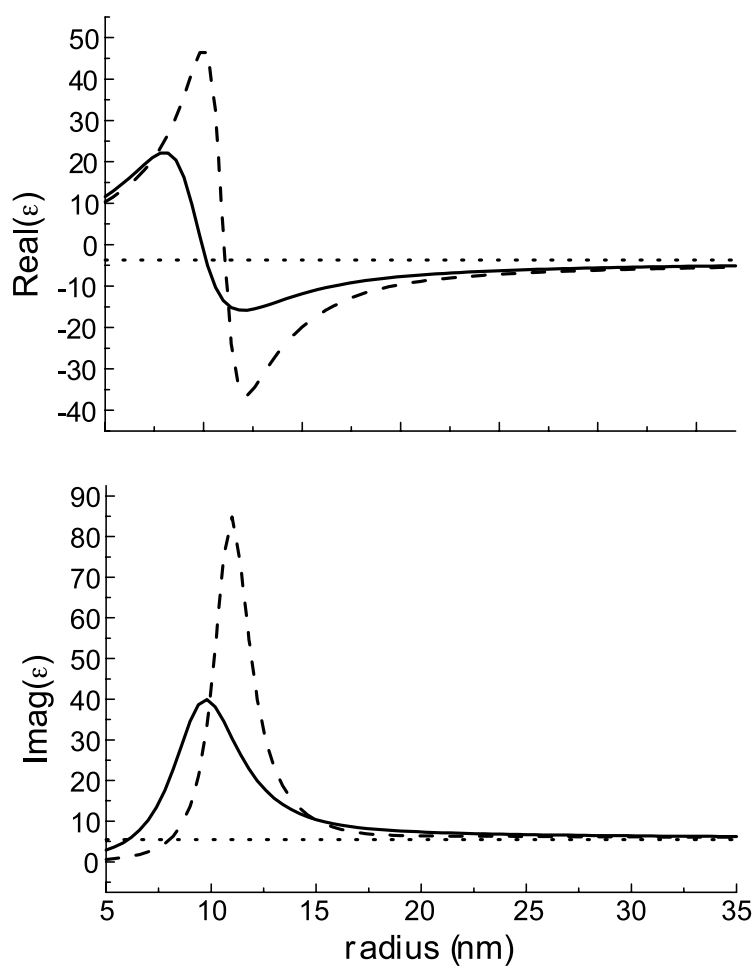

Fig. 8. Same as in Fig. 7, but for a higher metallic concentration $(f=$ $0.4)$ and the Bruggeman EMT has been applied. Note that the wavelength here is fixed at $\lambda=1.8 \mu \mathrm{m}$.

effect [12], and the surface scattering effect [12,13]; the present effect arises mainly from the wave nature of the surface electrons, leading to significant nonlocal dielectric response from the metal particle when its surface-to-volume ratio increases as a result of decrease in size. We believe the present result has significant implication to the comparison of various theories with experiments, in any optical study of composite materials with metallic nanoparticles. In particular, we have shown that, even in the Bruggeman EMT, resonant absorption can occur in a composite with large metallic concentration if the present nonlocal size-dependent effect is taken into account for particles with sizes in the order of a few nanometers.

\section{Acknowledgements}

This research is supported by National Science Council,
Taiwan, under grants numbers NSC-93-2112-M-019-004; NSC-93-2112-M-019-002; NSC-93-2120-M-019-001. One of us (PTL) also wants to acknowledge the support of the faculty development committee of Portland State University.

\section{References}

[1] J.C. Mawell-Garnett, Philos. Trans. R. Soc. London 203 (1904) 385; J.C. Maxwell-Garnett, Philos. Trans. R. Soc. London 205 (1906) 237.

[2] D.A.G. Bruggeman, Ann. Phys. (Leipzig) 24 (1935) 636.

[3] For a summary of earlier modifications, see R. Landauer, in: J.G. Garland, D.B. Tanner (Eds.), Electrical Transport and Optical Properties of Inhomogeneous Media, AIP, New York, 1978, pp. 2-43. (and references therein); A more recent review has been published by V.M. Shalaev, Phys. Rep., 272 (1996) 61.

[4] R.W. Cohen, G.D. Cody, M.D. Coutts, B. Abeles, Phys. Rev. B 8 (1973) 3689.

[5] C.G. Granqvist, J. Phys. (Paris) Colloq. 42 (1981) C1-247.

[6] P.M. Hui, D. Stroud, Phys. Rev. B 33 (1986) 2163.

[7] M. Gomez, L. Fouseca, G. Rodriguez, A. Velazquez, L. Cruz, Phys. Rev. B 32 (1985) 3429.

[8] M.F. MacMillan, R.P. Devaty, Phys. Rev. B 43 (1991) 13838.

[9] J.S. Ahn, K.H. Kim, T.W. Noh, D.H. Riu, K.H. Boo, H.E. Kim, Phys. Rev. B 52 (1995) 15244.

[10] V. Yannopapas, A. Modinos, N. Stefanou, Phys. Rev. B 60 (1999) 5359.

[11] R. Ruppin, Opt. Commun. 182 (2000) 273.

[12] D.M. Wood, N.W. Ashcroft, Phys. Rev. B 25 (1982) 6255.

[13] G.A. Niklasson, C.G. Granqvist, J. Appl. Phys. 55 (1984) 3382.

[14] R.G. Barrera, R. Fuchs, Phys. Rev. B 52 (1995) 3256.

[15] R. Fuchs, R.G. Barrera, J.L. Carrillo, Phys. Rev. B 54 (1996) 12824.

[16] C.I. Mendoza, R.G. Barrera, R. Fuchs, Phys. Rev. B 57 (1998) 11193.

[17] R. Fuchs, F. Claro, Phys. Rev. B 35 (1987) 3722.

[18] A rather comprehensive discussion of the different quantummechanical dielectric functions can be found in G.D. Mahan, Many-Particle Physics, 2nd ed., Plenum, New York, 1990.

[19] B. Abeles, J.I. Gittleman, Appl. Opt. 15 (1976) 2318.

[20] J.I. Gittleman, B. Abeles, Phys. Rev. B 15 (1977) 3273.

[21] See, e.g. T. Stephen Sathiaraj, R. Thangaraj, J. Phys. D: Appl. Phys. 30 (1997) 769.

[22] See, e.g. R. Ruppin, Phys. Rev. B 11 (1975) 2871; B.B. Dasgupta, R. Fuchs, Phys. Rev. B 24 (1981) 554; P.T. Leung, Phys. Rev. B 42 (1990) 7622.

[23] See the paper by V.M. Shalev cited in Ref. [3]. 